\title{
De Proyecto INCODE a FINCODA: utilización del Barómetro INCODE en alumnos universitarios $y$ en empleados con competencias de innovación.
}

Aznar-Mas, Lourdes Emilia ${ }^{a}$, Montero-Fleta, Begoñab, Pérez Peñalver, María Joséc, Watts, Frances $^{d}$, García-Carbonell, Amparo ${ }^{e}$, Marin-Garcia, Juan Antonio ${ }^{f}$

aEICE IEMA, Dpto. Lingüística Aplicada, UPV, laznar@idm.upv.es, b'EICE IEMA, Dpto. Lingüística Aplicada, UPV, bmontero@idm.upv.es, 'EICE IEMA, Dpto. Matemática Aplicada, UPV, mjperez@mat.upv.es, dEICE IEMA, Dpto. Lingüística Aplicada, UPV, fwatts@upv.es, ${ }^{\mathrm{e} E I C E ~ I E M A, ~}$ Dpto. Lingüística Aplicada, UPV, agarciac@upvnet.upv.es, fEICE IEMA, Dpto. Organización de Empresas, UPV, jamarin@omp.upv.es

\begin{abstract}
This paper reports on a project which emerged from the success of the European Project INCODE (Innovation Competencies Development), where the UPV Innovation Team EICE IEMA (Innovación en la Evaluación para la Mejora del Aprendizaje Activo), together with teams from other three European universities, presented an assessment tool for core competencies of innovation in university studies; a tool which was validated: the INCODE Barometer.

The present research is aimed at the adaptation of the INCODE Barometer for its use in companies and organizations, now within the European Project FINCODA (Framework for Innovation Competencies Development and Assessment) where the partner universities of the CARPE network (Consortium on Applied Research and Professional Education) including the $U P V$, and nine small, medium-sized and large companies are participating. EICE IEMA is an interdisciplinary group with members from six different Departments representing the UPV in this new project, in which the INCODE Barometer will be refined taking into account the outcomes of the needs analysis performed in the innovative companies selected.
\end{abstract}

Keywords: Innovation competencies, Assessment, Innovation in companies, Rubrics 
De Proyecto INCODE a FINCODA: utilización del Barómetro INCODE en alumnos universitarios y en empleados con competencias de innovación

\begin{abstract}
Resumen
Esta comunicación parte del éxito del Proyecto Europeo INCODE (Innovation Competencies Development) en el que el equipo de innovación IEMA de la UPV (Innovación en la Evaluación para la Mejora del Aprendizaje Activo), junto a equipos de otras tres universidades europeas, presentó como resultado un instrumento de evaluación de las competencias genéricas de innovación en el ámbito universitario. Instrumento que ya ha sido probado y validado: el Barómetro INCODE.
\end{abstract}

La novedad de la investigación actual es la adecuación del Barómetro INCODE al ámbito de la empresa, en el marco del proyecto FINCODA (Framework for Innovation Competencies Development and Assessment). En este proyecto participan cinco universidades de CARPE (Consortium on Applied Research and Professional Education), del que la Universitat Politècnica de València (UPV) es socia, junto a nueve pequeñas, medianas y grandes empresas. El grupo de innovación IEMA, equipo multidisciplinar con profesores de seis departamentos, representa a la UPV en este proyecto de desarrollo, en el que se llevará a cabo el refinamiento del Barómetro INCODE a partir del análisis de necesidades de empresas innovadoras.

Palabras clave: Competencias de Innovación, Evaluación, Innovación en la Empresa, Rúbricas

\title{
1. Introducción
}

El contexto socio-económico actual presenta retos para las empresas y organizaciones en las que únicamente un buen producto no garantiza el éxito de un buen posicionamiento en el mercado. Los estándares de Investigación, Desarrollo e Innovación están cambiando y giran en torno a otras variables que destacan a unas organizaciones frente a otras. En el entorno del mundo globalizado en el que vivimos, sólo las nuevas ideas marcarán la diferencia (García, 2012). Tenemos ante nosotros diversos factores que debemos tener en cuenta y resultados que debemos analizar y meditar. La innovación es un eje sobre el que no sólo se genera productividad y mayor crecimiento, también mejora el comportamiento de algunas empresas y les lleva a ser un referente de impacto.

Esos nuevos resultados surgen como consecuencia de la experimentación, de cambios significativos que se producen en un determinado contexto en un momento dado; de la capacidad de crear, del aprendizaje a través de la interacción entre personas. Estos resultados en la empresa se transforman en resultados llamativos. 
La capacidad de innovación es un valor cada vez más en alza para la formación de los alumnos universitarios y para las empresas, que se suman a un cambio de paradigma para responder mejor a las necesidades que la sociedad demanda. El mundo profesional ha dado un vuelco y requiere de empresas sostenibles a través de la innovación. Para ello, la empresa necesita una respuesta rápida de su personal, que contribuirá a ese cambio con la actitud adecuada. Estamos en un momento en que la experiencia se comparte y en la que no hay innovación posible si no hay una percepción real de la misma. Es esencial que se produzca el desarrollo adecuado de destrezas, de competencias esenciales para el desarrollo individual y la competitividad, como indica el Marco Europeo de Cualificaciones para el aprendizaje permanente (European Commission, 2008).

Para alcanzar el reto expuesto es necesario que el contexto sea dinámico, que las empresas tengan un compromiso de formación de su personal para la adaptación a nuevos esquemas organizativos u otro tipo de prácticas. También es necesaria una mejor gestión del capital humano y del talento, analizando las diferentes categorías laborales (Marin-Garcia et al., 2011) . Cada individuo posee una serie de habilidades que le permiten actuar de una forma inteligente, utilizando los recursos que tiene a su disposición si se le prepara para ello; lo que podría revelar su competencia de innovación. Es por ello que en este trabajo se intenta consensuar una serie de indicadores que revelen el comportamiento innovador del personal de empresa. En definitiva, una empresa que está dispuesta a aprender y a cambiar será más proclive a ser innovadora y sostenible.

Hasta ahora, cualquier empresa debía mantener un vínculo estrecho con el mercado para ser eficaz y productiva. Actualmente se valora que la empresa esté estrechamente relacionada con otras entidades para poder implementar con éxito el proceso de innovación: laboratorios, universidades, legisladores, entre otras. La coordinación activa entre empresas o con instituciones públicas que investiguen sobre este proceso tan complejo es necesaria (OECD/Eurostat, 2005). De ahí que nuestro trabajo se centre en la relación entre universidades que poseen un sistema de formación de calidad y una selección de empresas innovadoras.

El Proyecto INCODE (Innovation Competencies Development 518132-LLP-1-2011-1-FIERASMUS-FEXI) tuvo una duración de dos años desde 2011 hasta 2013 y se llevó a cabo con financiación del Life-long Learning Programme de la Unión Europea. Su objetivo principal era el de crear un instrumento para medir los resultados de los alumnos que desarrollaran su aprendizaje en un entorno donde se generaban diferentes capacidades. Dichas capacidades llevaron a la definición de la competencia de innovación. Aunque en la mayoría de los casos la adquisición de habilidades, conocimientos y actitudes se infiere pero no se evalúa adecuadamente, incluso cuando se incluye como parte de la evaluación sumativa (Watts et al., 2012). Las empresas y organizaciones que empleen a dichos 
De Proyecto INCODE a FINCODA: utilización del Barómetro INCODE en alumnos universitarios y en empleados con competencias de innovación

alumnos, tendrán entre su personal a personas mejor cualificadas para implicarse en procesos innovadores en sus lugares de trabajo.

El instrumento Barómetro INCODE fue probado y validado (Watts et al., 2013).

\section{Objetivos}

El Proyecto FINCODA (Framework for Innovation Competencies Development and Assessment 554493-EPP-1-2014-1-FI-EPPKA2-KA) nace del proyecto INCODE. Comenzó en enero de 2015 y tiene una duración hasta diciembre de 2017. FINCODA se centra en dos de las prioridades del marco estratégico para la cooperación europea en el ámbito de la educación y la formación (ET 2020, 2009): el objetivo estratégico número 2 (mejorar la calidad y la eficacia de la educación y de la formación) y el objetivo estratégico número 4 (incrementar la creatividad y la innovación, incluido el espíritu empresarial, en todos los niveles de la educación y la formación).

Los principales objetivos de FINCODA son:

- Identificar los comportamientos, conocimientos y destrezas innovadores en la empresa y relacionarlos con los de nuestros alumnos.

- Observar los comportamientos, conocimientos y destrezas innovadores tanto en el ámbito docente universitario y en el de la empresa a través de un instrumento común de evaluación, el Barómetro INCODE, a nivel nacional como internacional. Es vital que universidad y empresa trabajen en paralelo para poder medir los resultados que se persiguen.

- Revisar el Barómetro INCODE para adecuar su uso en la empresa y que se convierta en un instrumento para el desarrollo e implementación de innovación tanto en enseñanza superior como en la empresa.

En el proyecto FINCODA participan cinco universidades europeas: TUAS (Turku University of Applied Sciences) de Finlandia; HAW (Hamburg University of Applied Sciences) de Alemania; HU (Utrecht University of Applied Sciences) de los Países Bajos; MMU (Manchester Metropolitan University) del Reino Unido y la UPV (Universitat Politècnica de València) de España. Todas las universidades que participan en el proyecto FINCODA pertenecen a la red CARPE (Consortium on Applied Research and Professional Education). Estas universidades aportan experiencia, entre otras áreas, en gestión de proyectos internacionales sobre educación universitaria, metodología de evaluación basada en el comportamiento y análisis psicométricos, entre otros. Sin embargo, lo que confiere especial originalidad al proyecto FINCODA es la participación, además de estas universidades, de nueve empresas europeas que desarrollan e implementan innovación y que trabajan con personal innovador. Las nueve empresas que participan son: Elomatic Ltd. 
(Finlandia); Hamburger Hafen und Logistik AG (Alemania); Lactoprot (Alemania), ECDL (Holanda); John Caunt Scientific Ltd. (Reino Unido); Carter \& Corson Partnership Ltd (Reino Unido); Enterprise Europe Northwest (Reino Unido); Celestica (España) y Schneider Electric España S.A. (España).

FINCODA está liderado por un director de proyecto, responsable de la coordinación, que es la persona de contacto con la EACEA (Education, Audiovisual and Culture Executive Agency) de la Unión Europea. También existe un Comité de Gestión del Proyecto, integrado por un representante de cada institución participante en calidad de socio. Respecto a la distribución del trabajo de investigación, se establece de acuerdo con la experiencia e interés de los miembros de las instituciones participantes en cada tema. Cada universidad lidera un paquete de trabajo afín a su perfil investigador; la gestión del proyecto se hace desde la universidad, y las empresas participantes se concentran junto al personal investigador de las instituciones académicas en el contenido de los paquetes de trabajo.

\section{Desarrollo de la innovación}

La metodología de FINCODA se materializa en las siguientes acciones para alcanzar los objetivos previstos:

- Identificar las necesidades y estado de la cuestión del desarrollo de competencias de innovación y evaluación en las empresas participantes.

- Investigar sobre métodos novedosos para la identificación de personas innovadoras.

- Refinar el Barómetro INCODE y adaptarlo a las nuevas necesidades que surgen de esta investigación.

- Crear el software de aplicación adecuado para la utilización del nuevo Barómetro INCODE.

- Preparar los instrumentos a nivel profesional para la evaluación del comportamiento innovador.

- Formar al personal de las organizaciones participantes para que evalúen el comportamiento innovador.

- Validar la nueva versión del Barómetro INCODE tras probarlo en las organizaciones participantes.

- Analizar los resultados de la validación y ajuste del barómetro.

- Difundir a gran escala de los resultados del proyecto.

Con respecto a la secuenciación del trabajo se prevé una actuación permanente durante los próximos tres años por parte de los socios participantes, a la que se añade un buen número

(cc)) EY-NC-ND 2015, Universitat Politècnica de València

Congreso In-Red (2015) 
De Proyecto INCODE a FINCODA: utilización del Barómetro INCODE en alumnos universitarios y en empleados con competencias de innovación

de reuniones para análisis y puesta en común del trabajo realizado y de los resultados obtenidos. A continuación se presentan algunas de las fechas más relevantes:

- Mes 1. Reunión de lanzamiento, donde se redefine el plan inicial del Proyecto a partir de la evaluación de la propuesta y de los cambios sugeridos por las organizaciones participantes.

- Mes 4. Análisis de necesidades en las empresas participantes. Los resultados orientan el desarrollo de las actividades futuras hacia los elementos que proporcionen valor añadido para las empresas.

- Mes 8. Primera versión piloto del software para el Barómetro INCODE. La aplicación se ha de someter a pruebas antes del proceso de validación del barómetro en las respectivas universidades y empresas participantes.

- Mes 13. Nueva versión del Barómetro INCODE y taller de formación de evaluadores; éste es un paso crucial para las siguientes fases de trabajo del proyecto.

- $\quad$ Mes 13. Software para el Barómetro INCODE: tras haber sido sometido a pruebas con grupos piloto de estudiantes y haber hecho las mejoras necesarias después de la evaluación de su feedback.

- Mes 14. Lanzamiento de la validación del Barómetro INCODE. Pruebas piloto en universidades y empresas proporcionan la información para la validación.

- Mes 17. Auditoria/evaluación provisional del proceso de validación y de los pilotajes, que proporciona información de cómo proceder con los pilotajes y si hay información suficiente para la validación.

- Mes 23. Actualización del plan para la diseminación y explotación: actualización del plan de valoración y comienzo de la implementación a gran escala de las actividades de valoración.

- $\quad$ Mes 26. Lanzamiento de cursos MOOC. Uno sobre evaluación de comportamiento y otro sobre la utilización de la herramienta de evaluación de la competencia de innovación.

- Mes 30. Auditoria/evaluación provisional de la diseminación y explotación: análisis del feedback de actividades de valorización y re-definición del plan para posterior valorización.

- Mes 34. Análisis final de los resultados y plan para futuras actuaciones.

\section{Resultados}

Los resultados principales previstos del proyecto FINCODA son unos productos que sean útiles tanto para universidades como para empresas que están implicadas en la innovación. Los resultados en forma de recursos educativos son, por ejemplo, una aplicación (software)

(c)) EY-NC-ND 2015, Universitat Politècnica de València

Congreso IN-RED (2015) 
para la evaluación de las competencias de innovación, que estará en línea y servirá tanto para la auto-evaluación como para la evaluación entre pares y por el profesor. Otro de los productos será un programa de formación de evaluadores con cursos tipo MOOC para la evaluación del comportamiento innovador.

Actualmente el proyecto FINCODA se encuentra en sus comienzos, en la fase de análisis de necesidades, que incluye encuestas por correo electrónico a un amplio abanico de empresas, la búsqueda de las herramientas que existen en el mercado y entrevistas con las empresas socias del proyecto. Aunque está pendiente de concluir el periodo de recepción de las encuestas, ya se ha llevado a cabo una búsqueda de herramientas (cuestionarios) basadas tanto en el objeto como en el sujeto, a nivel de organización, grupo e individuo. Se han encontrado dos herramientas de interés: GISAT 2.0 e INNDUCE.me. Un taller sobre innovación, capacidades y detección de necesidades llevado a cabo con los socios participantes en la segunda reunión del proyecto, celebrada en Manchester en mayo 2015, también forma parte de la fase inicial.

GISAT 2.0, promocionado por The Conference Board of Canada, puede usarse en el reclutamiento de personal al ser una prueba de aptitud general en las destrezas de innovación. INNDUCE.me ha sido ideado por la Universidad de Gante después de la revisión sistemática de más de 6.000 trabajos, en la que se vio la relación entre el comportamiento relacionado con la innovación y el éxito. Es una herramienta que puede ser utilizada por empleadores, organizaciones y empleados. En la revisión de la literatura se identificaron cuatro dimensiones de comportamiento laboral innovador (Innovative Work Behavior): generar ideas innovadoras, explorar estas ideas, recabar apoyo y recursos para la realización de las ideas innovadoras y poner en práctica las ideas innovadoras.

De las dos entrevistas preliminares realizadas hasta el momento a empresas socias de FINCODA destaca que la innovación es la transformación de la creatividad en algo práctico y tangible, así como la creencia de que todo el mundo puede ser innovador. Las empresas resaltaron que las limitaciones económicas, de tiempo y de practicidad actúan como freno a la innovación. El propósito de las entrevistas era obtener los puntos de vista y experiencias de empresas conocidas por su innovación, en relación a las competencias de innovación que buscan para su plantilla y cómo las identifican. Las empresas constataron su deseo de que FINCODA elaborase una herramienta que detallase tanto el estilo innovador del candidato para trabajar en su empresa, como el entorno que puede obtener lo mejor de los empleados.

Los resultados del taller realizado con las nueve empresas se obtuvieron a partir de los comentarios hechos a cada uno de los veinticinco indicadores del Barómetro INCODE, que se clasifican en tres dimensiones: competencias individuales, competencias interpersonales y networking. Se proporcionaron algunos ejemplos de comportamiento observable aplicado al contexto laboral. Desde la perspectiva empresarial, comprender las necesidades del

\section{(c)) EY-NC-ND 2015, Universitat Politècnica de València}


De Proyecto INCODE a FINCODA: utilización del Barómetro INCODE en alumnos universitarios y en empleados con competencias de innovación

cliente se estimó significativo en relación a las competencias individuales de innovación, siendo el enfoque en el cliente de especial importancia cuando se presentan nuevos modos de implementación de ideas y se consideran las tareas desde distintos puntos de vista. Al hablar de las competencias interpersonales, los socios resaltaron las diferentes culturas y prácticas organizativas en empresas europeas. Los socios alemanes, por ejemplo, hicieron hincapié en la naturaleza jerárquica en el trabajo en su país y la formalidad de las relaciones entre los mandos y los trabajadores. Los socios finlandeses, por otro lado, subrayaron la naturaleza abierta e igualitaria de las relaciones laborales en su país. Por tanto, factores de contexto de la organización son determinantes para iniciar y llevar a cabo la innovación. Ejemplos de los factores son la actitud ante el riesgo, el tamaño de la empresa y los niveles de autonomía individual.

Las implicaciones para FINCODA sugieren que la herramienta para medir las competencias individuales de innovación en un entorno empresarial ha de tener en cuenta la etapa en la que se encuentra el individuo en su carrera profesional, su posición en la empresa y el papel de su puesto de trabajo. Una consideración adicional es el fin con el que se pretende utilizar el barómetro. En términos de reclutamiento, desde la perspectiva empresarial, se podría distinguir entre reclutamiento general y otro que tenga en cuenta un nivel de estudios superiores. Si la herramienta se usa en la formación y desarrollo del empleado, la etapa profesional del individuo puede ser importante para determinar las competencias de innovación que busca la empresa. Las empresas recalcaron la necesidad de una herramienta flexible y adaptable.

El segundo punto concluyente del taller trata del alcance de proyecto FINCODA. El objetivo del proyecto es desarrollar una herramienta para medir las competencias de innovación del individuo, no de los equipos o de la cultura organizativa. Sin embargo, desde la perspectiva empresarial, las estructuras y culturas organizativas juegan un papel importante en la determinación del tipo de comportamientos innovadores del individuo, que se estiman apropiados y efectivos para impulsar la innovación.

\section{Conclusiones}

Investigar cómo se generan los procesos en los que se producen innovaciones es un objetivo crucial en el futuro europeo y global. Las empresas innovadoras pueden jugar un papel importante en el conocimiento de tales procesos y en la puesta en marcha de instrumentos que permitan medir y a la vez explicitar pautas de comportamiento relacionadas con la innovación. Para ello, la formación adecuada de sus mandos a la hora de evaluar la competencia de innovación de su personal es fundamental.

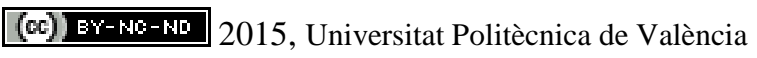

Congreso IN-RED (2015) 
Es necesaria, asimismo, una mayor colaboración y acuerdos entre universidad-empresa que favorezcan la provisión de un marco de empleabilidad a través de la innovación. La universidad se tiene que convertir en el motor del cambio, implementando una evaluación de competencias de innovación que sea fiable y eficaz (Pérez Peñalver et al., 2012). Esos resultados deben ser también transferidos a las empresas implicadas en innovación.

El refinamiento del Barómetro INCODE, teniendo en cuenta la experiencia previa, la bibliografía sobre el tema y el punto de vista del sector empresarial llevará a una mejora del propio instrumento, así como a la validación del mismo para contextos distintos del universitario. A su vez, la creación de un programa de formación, herramientas en línea, software y cursos en abierto para el uso del instrumento permitirá su difusión a nivel internacional, así como la toma de datos respecto a su utilización.

\section{Agradecimientos}

Este trabajo ha sido realizado con la financiación del programa Erasmus+ de la Unión Europea: FINCODA Framework for Innovation Competencies Development and Assessment 554493-EPP-1-2014-1-FI-EPPKA2-KA (The European Commission support for the production of this publication does not constitute an endorsement of the contents which reflects the views only of the authors, and the Commission cannot be held responsible for any use which may be made of the information contained therein).

\section{Referencias}

CARPE. Consortium on Applied Research and Professional Education. http://husite.nl/carpenetwork [Consulta: 22 de mayo de 2015]

CONCLUSIONES DEL CONSEJO, de 12 de mayo de 2009, sobre un marco estratégico para la cooperación europea en el ámbito de la educación y la formación (ET 2020) [Diario Oficial de la Unión Europea, C 119, 28.5.2009, pp.2-10].

EUROPEAN COMMISSION (2008). The European Qualifications Framework for Lifelong Learning (EQF). Luxembourg: Office for Official Publications of the European Communities.

GARCÍA, S. (2012). "La empresa que aprende” en Savia. Revista de economía y gestión de viajes. Época V, febrero, $\mathrm{n}^{\circ}$ 7, pp.52-53.

INCODE. Innovation Competencies Development. <http://www.incode-eu.eu/en> [Consulta: 20 de mayo de 2015]

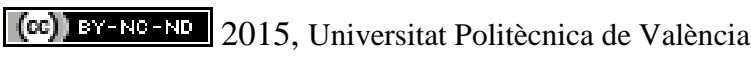

Congreso In-Red (2015) 
De Proyecto INCODE a FINCODA: utilización del Barómetro INCODE en alumnos universitarios y en empleados con competencias de innovación

INNDUCE.ME <http://www.innduce.me/en\#what> [Consulta: 20 de mayo de 2015]

MARIN-GARCIA, J. A.; AZNAR-MAS, L. E. y GONZÁLEZ LADRÓN DE GUEVARA, F. (2011) "Innovation types and talent management for innovation" en Working Papers on Operations Management, Vol. 2, n 2, pp. 25-31.

OECD/Eurostat (2005), Oslo Manual: Guidelines for Collecting and Interpreting Innovation Data, 3rd Edition, The Measurement of Scientific and Technological Activities, Paris: OECD Publishing.

PÉREZ PEÑALVER, M. J, AZNAR-MAS, L.; WATTS, F. (2012). “To adapt or to die when leaving the university: To promote innovation competence may be the key” en INTED Proceedings, pp. 27312736.

RECOMENDACIÓN DEL PARLAMENTO EUROPEO Y DEL CONSEJO de 23 de abril 2008 relativa a la creación del Marco Europeo de Cualificaciones para el aprendizaje permanente. [Diario Oficial de la Unión Europea C111, 6.05.2008, pp. 1-7].

THE CONFERENCE BOARD OF CANADA. Education and Learning. General Innovation Skills Aptitude Test (GISAT 2.0)

http://www.conferenceboard.ca/topics/education/learning-tools.aspx. [Consulta: 20 de mayo de 2015]

WATTS, F., MARIN-GARCIA, J. A., GARCÍA-CARBONELL, A. y AZNAR-MAS, L. E. (2012). "Validation of a rubric to assess innovation competence" en Working Papers on Operations Management, Vol. 3, n 1 , pp. 61-70

WATTS, F., GARCÍA-CARBONELL, A. y ANDREU ANDRÉS, M. A. (eds.) (2013) Innovation competencies development: INCODE Barometer and user guide. Turku: Turku University of Applied Sciences. 